\title{
Device Risk Code
}

National Cancer Institute

\section{Source}

National Cancer Institute. Device Risk Code. NCI Thesaurus. Code C95360.

A coded value specifying the risk associated with the device. 